Retraction

\title{
Retraction Note: Cromakalim, a Potassium Channel Opener, Ameliorates Organophosphate- and Carbamate-Induced Seizures in \\ Mice
}

\author{
Zahid Hussain Khan ${ }^{1, *}$ \\ ${ }^{1}$ Department of Anesthesiology and Intensive Care Imam Khomeini Medical Center, Tehran University of Medical Sciences, Tehran, IR Iran \\ "Corresponding author: Zahid Hussain Khan, Department of Anesthesiology and Intensive Care Imam Khomeini Medical Center, Tehran University of Medical Sciences, Tehran, \\ IR Iran. Tel: +98-2161192828, Fax:+98-2166938885, E-mail: khanzh51@yahoo.com \\ Received 2018 March 03; Accepted 2018 March 03. \\ This retracts the article RETRACTED ARTICLE: Cromakalim, a Potassium Channel Opener, Ameliorates Organophosphate- and Carbamate-Induced Seizures in Mice.
}

Following the author's declaration received to the journal on Archives of Neuroscience (Ticket \#301440) and in line with COPE flowchart about duplicate publications, the editor-in-chief of the journal decided to retract the mentioned article from the publishing process. Based on a written document, the corresponding author (Ahmad-Reza Dehpour) declared that the duplicate submission was done by one of the authors (Sattar Ostadhadi).

Kind Regards

Editor-in-Chief

Prof. Zahid Hussain Khan 